\title{
ZOOBENTHOS ASSOCIATED WITH SUBMERGED MACROPHYTES IN LITTORAL AREAS OF LAKE VICO (ITALY): SOME RELATIONS BETWEEN FAUNA STRUCTURE AND WATER QUALITY
}

\author{
L. Mastrantuono \\ Department of Animal and Human Biology, University of Rome. Viale dell'Università 32, 00185 Roma. Italia.
}

Keywords: littoral zoobenthos, lakes, water quality.

\begin{abstract}
The invertebrate fauna associated with submerged macrophytes in some areas of Lake Vico was analysed following the intensification of agricultural practices in the surrounding land in the last decade. The fauna was qualitatively rich and quantitatively abundant. A total of 110 taxa were identified, widely distributed over all stations and quantitatively represented mainly by Crustacea, Nematoda and Chironomidae, and secondarily by several other groups accounting for quite high percentages. The analysis of structural parameters of the community indicated a satisfactory environmental quality in the zones considered. Some significant differences between stations pointed to the interest of qualitative and quantitative aspects of the community in the environmental monitoring of littoral lacustrine zones.
\end{abstract}

\section{INTRODUCTION}

The composition and distribution of the invertebrate fauna associated with submerged macrophytes was analysed in some littoral areas of Lake Vico, located close the cultivated lands surrounding the North part of the lake. The intensification of agricultural activities in the last decade pointed to the advisability of analysing the littoral biocoenosis in order to identify possible negative effects on littoral fauna, also considering the importance of the lake for fishing. Moreover, a study carried out in the same period and sites on the zoobenthos living in sandy shores (MASTRANTUONO \& LA ROCCA, 1988) showed concrete evidence of a highly trophic condition in the zones directly exposed to the effects of the cultivations (hazel orchards), periodically subjected to treatments with fertilizers and pesticides.

Previous data refer to multidisciplinary research on chemical and biological aspects of the lake

Limnetica. 7: 153-162 (1991)

(C) Asociación Española de Limnología, Madrid, Spain
(BARBAnti et al., 1971). At that time it was classified as mesotrophic and in natural conditions, supported by the absence of important pollution sources. Littoral and sublittoral zoobenthos have been studied (NocentinI, 1973) with reference only to some zoological groups (mainly Oligochaeta, Chironomidae and Gastropoda) and considering as a whole the fauna associated with bottoms and submerged vegetation.

So, this analysis had the purpose both to define, as completely as possible, the composition of the meio- and macrofauna associated with submerged macrophytes, and to verify the role of some qualitative and quantitative aspects of the community in an evaluation of environmental quality of littoral lacustrine zones.

Owing to the high heterogeneity of both the fauna and the substratum and to the remarkable environmental variations of physico-chemical parameters, this community has traditionally been considered less significant than the bottom community in evaluating water quality. So, several authors (PIECZYŃSKI, 1973; SosZKA, 1975; BIGGS \& Malthus, 1982; Brown et al., 1988) have adres- 
sed their attention principally to the relations between macrobenthic organisms and substratum. However, some indications referring to both invertebrates and macrophytes (OzIMEK \& SikoksKA, 1976; ClakKe, 1979; LaCHaVANNF. 1985; MasTRANTUONO, 1986, 1987) supplied useful elements to clarify the interest of the community in environmental monitoring.

\section{MATERIAL AND METHODS}

Lake Vico, located in a volcanic area at $510 \mathrm{~m}$ a.s.l., has a surface area of $12.081 \mathrm{~km}^{2}$. a perimeter of $16.9 \mathrm{~km}$ and a maximum depth of $48.5 \mathrm{~m}$. The water level is regulated by an effluent (Rio Vicano) artificially connected to the lake in the sixteenth century through an underground tunnel (fig. 1). This effluent caused the emersion of a wide part of land in the north of the lake. where the difference between the ancient shoreline and the present one is noteworthy (fig. 1, c). The lake is characterized by a small catchment-basin (40.93 $\mathrm{km}^{2}$ ) and by a rain water flow largely dispersed on the lands. A few small affluents, which lie to the $\mathrm{N}$ and $\mathrm{NE}$ of the lake (fig. 1. r), pass through the cultivated soils (B ARBANTI, 1969).

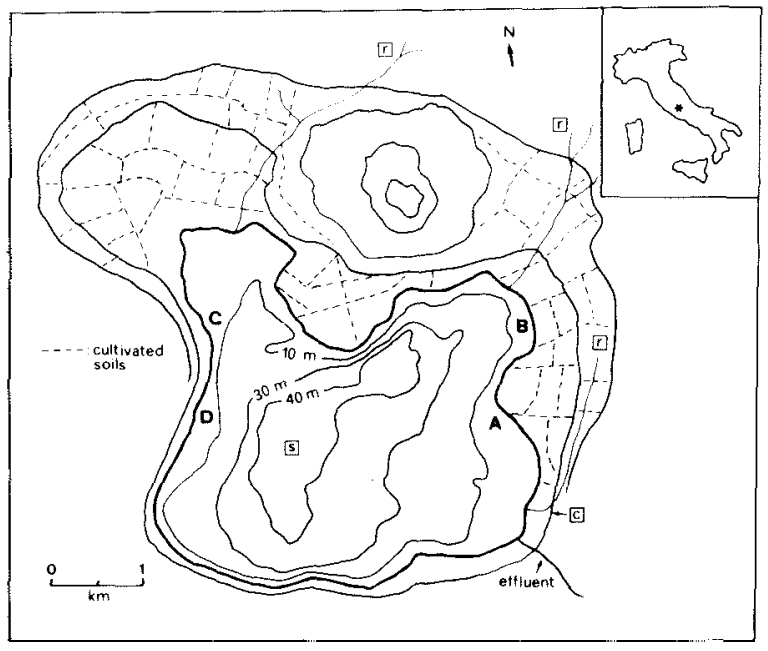

Figure 1.- Map of Lake Vico and location of the sampling stations (A. B, C, D).

Mapa del lago Vico y localización de los puntos de muestreo (A, B, C. D).
The lake is monomictic with summer stratification, the temperature varied from $6.5^{\circ} \mathrm{C}(\mathrm{Fe}$ bruary) to 25 "C (August) in surface waters and from 6.2 "C to 9 " $\mathrm{C}$ in deep waters. Oxygen, high in winter at all depths (about $10 \mathrm{mg} / \mathrm{l}$ ), showed a considerable depletion in summer below a depth of $30 \mathrm{~m}(0.2 \mathrm{mg} / \mathrm{l}) . \mathrm{pH}$ was alkaline (from 7.2 to 8.8 throughout the year) and the transparency was high (maximum value: $13 \mathrm{~m}$; annual mean: $6.5 \mathrm{~m}$ ). Small quantities of nutrients were observed in the water column 0-40 m (P total: $21 \mu \mathrm{g} / \mathrm{l}, \mathrm{N}-\mathrm{N} 03$ : 13 $\mu \mathrm{g} / \mathrm{l}, \mathrm{N}-\mathrm{NH} 3: 18 \mu \mathrm{g} / \mathrm{l}, \mathrm{N}-\mathrm{N} 02: 0.8 \mu \mathrm{g} / \mathrm{l}$, annual averages, station s, fig. 1), indicating an oligo-mesotrophic condition in the waters (O.E.C.D. report, 1982).

The samples of benthos were collected bimonthly from March 1985 to March 1986 in four sampling zones (A, B, C, D, fig. 1), chosen in areas differently influenced by the surrounding cultivations. Stations A and B were located close to the cultivated land, station $\mathrm{C}$ was farther away and station $\mathrm{D}$ was at a considerable distance from the cultivations. The sampling was carried out for each station and date at three depth intervals (0-3 $\mathrm{m}, 3-6 \mathrm{~m}, 6-10 \mathrm{~m}$ ) using a sledge dredge (opening: $35 \mathrm{~cm}$ in width, $20 \mathrm{~cm}$ in height, mesh size: 180 $\mu \mathrm{m})$. It was dragged for about $50 \mathrm{~m}$, parallel to the shore, following a sinusoidal path at the selected depth interval. The material was preserved in $10 \%$ formalin. In the laboratory the plants were washed and separated from all invertebrates. The macrobenthic organisms were sorted from the whole sample and the meiobenthic ones were counted in four subsamples that, together, represented 1116 of the entire sample.

The similarity between stations was evaluated using the qualitative QS coefficient (SøRENSEN, 1948) and the quantitative PSc index (RENKONEN, 1938). Community structure was analysed by means of the Shannon index (MARGalef, 1957) and evenness index (Pielou, 1966).

\section{RESULTS}

\section{Submerged macrophytes}

A total of 13 taxa were identified (table 1), whose distribution reached a maximum depth of about 
Table I.- Submerged macrophyte composition in Lake Vico: seasonal variations and bathymetric distribution. $+=$ present; $o=$ rare; $\bullet$ absent: +++ abundant.

Composición de los macrófitos sumergidos del lago Vico: variaciones estacionales y distribución batimétrica. $+=$ presente; $0=$ rara: $\bullet$ auscnte; +++ abundante.

\begin{tabular}{|c|c|c|c|c|c|c|c|c|c|c|}
\hline & \multicolumn{5}{|c|}{1985} & \multicolumn{2}{|c|}{1986} & \multicolumn{3}{|c|}{ Depth interval $(m)$} \\
\hline & 111 & V & V11 & $I X$ & $X I$ & I & III & $0-3$ & $3-6$ & $6-10$ \\
\hline \multicolumn{11}{|l|}{ Halorrhaginaccae } \\
\hline Myriophyllum spicatum & $a$ & $a$ & +++ & +++ & +++ & +++ & • & + & + & a \\
\hline \multicolumn{11}{|l|}{ Polygonaceac } \\
\hline Polygonum amphyhrum & - & - & $\circ$ & 0 & $\bullet$ & - & - & + & - & - \\
\hline \multicolumn{11}{|l|}{ Najadaceae } \\
\hline Najm marina & - & 0 & 0 & 0 & - & - & - & + & + & + \\
\hline Najm minor & - & - & o & 0 & - & - & - & + & + & + \\
\hline \multicolumn{11}{|l|}{ Ranunculaceae } \\
\hline Ranunculus sp & - & - & - & 0 & - & - & - & + & - & - \\
\hline \multicolumn{11}{|l|}{ Potamogetonaceae } \\
\hline Potarnogeton pectinatus & - & - & $+t+$ & $t+t$ & $t+t$ & +++ & - & - & + & + \\
\hline Potamogeton pusillus & - & - & $a$ & $\circ$ & - & • & - & + & - & - \\
\hline Potarnogetonlucens & - & - & $\circ$ & 0 & - & - & - & + & - & - \\
\hline Potamogefon crispus & - & - & 0 & 0 & $\circ$ & - & - & + & - & • \\
\hline \multicolumn{11}{|l|}{ Characeae } \\
\hline Chara sp. a & - & - & +++ & $++t$ & +++ & - & - & + & + & + \\
\hline Chara sp. b & - & - & $\circ$ & $\bullet$ & $\bullet$ & - & - & + & - & - \\
\hline Nitella sp. & - & - & 0 & - & - & - & - & + & - & - \\
\hline \multicolumn{11}{|l|}{ Ceratophyllaceae } \\
\hline Ceratophyllum demersum & $++t$ & +++ & +++ & $++t$ & +++ & $++t$ & +++ & - & + & + \\
\hline
\end{tabular}

$12 \mathrm{~m}$, in relation to the high transparency values. The vegetation was quantitatively quite abundant and reached its maximum species richness and quantitative presence in summer. The dominant taxa were Myriophillum spicatum, Potamogeton pectinatus, Chara sp. and Ceratophyllum demersum. This latter, always present during the year mainly at depths from 6 to $10 \mathrm{~m}$, covered a good proportion of the littoral bottoms.

The macrophyte composition, according to categories proposed by Lachavanne (1985), can be considered indicative of a mesotrophic condition. This evaluation is based on the following observations: a) medium-high level of species richness; b) colonization to a depth of $10-12 \mathrm{~m}$; c) association of taxa typical of better environmental conditions (Ranunculus sp., Chara sp.) with others tolerant the organic increase (P.pectinatus, $\mathrm{P}$. pusillus).

\section{Invertebrate fauna}

The fauna included a total of 110 taxa belonging principally to Chironomidae (larvae), Oligochaeta and Cladocera, secondarily to Nematoda, Hydracarina, Gastropoda and Copepoda.
Crustacea, mainly Copepoda, were quantitatively predominant in all stations, followed by $\mathrm{Ne}$ matoda and Chironomidae with lower percentages. Several other groups such as Oligochaeta, Gastropoda, Hydracarina, Turbellaria and Hydroida were present in comparatively high percentages at least in some stations (table 2).

Copepoda reached very high abundance mainly at stations A, B and C (range: 60.4-67.1\%). The dominant species were Macrocyclops albidus, Eucyclops serrulatus and Eucyclops macruroides (table 3), typical cyclopids of littoral zones, having a large geographical diffusion and a wide ecological range. The remarkable occurrence of pelagic cyclopids (Cyclops abyssorum and $\mathrm{Me}$ socyclops leuckarti) represents a phenomenon already observed in Lake Nemi and in Lake Campotosto (Mastrantuono, 1986, 1987), and probably related to high mobility and particular biological cycles of these species.

Cladocera were composed of a high number of species (12) most of which attained a considerable percentage at all stations. Acroperus harpae, Chydorus sphaericus, Alona affinis, Simocephalus vetulus and Eurycercus lamellatus represented the 
dominant cladocerans. Other crustaceans (Ostracoda, Isopoda and Amphipoda) displayed a sporadic presence, while an uncommon abundance of the decapod Palaemonetes antennarius, disappeared from some lakes in Central Italy, was observed everywhere.

Among insects, Chironomidae constituted the most diversified and abundant group, although with moderate percentages. Psectrocladius psilopterus, Tanytarsus, Labrundinia and Larsia reached the highest percentages, while Chironominae had generally low presence. All chironomid taxa previously recorded (Nocentini, 1973) were found, and also some others belonging to Pentaneurini, Orthocladiinae and Chironominae. Other insects showed usual percentages for Central Italy (Lake Nemi, Lake Campotosto, Lake Albano), with the exception of Ephemeroptera, present in Lake Vico with higher number of taxa (6) and percentage values (range: 0.2-1.0\%).
Nematoda, comprising 8 taxa, were mostly represented at all stations by Ethmolaimus pratensis, a common nematode in Italy. High abundances of nematodes have been observed at station $\mathrm{D}$ $(12.5 \%)$ where also D. asymphydorus reached considerable percentages $(5 \%)$.

Oligochaeta were present with a higher number of taxa (19) and lower percentage values than in other studied lakes in Central Italy. Only at station A were relatively high percentages of oligochaetes $(9.4 \%)$ were found, due to a conspicuous abundance of Nais variabilis (4.6\%), Nais simplex $(3.4 \%)$ and Nais communis (1.2\%).

Hydracarina and Gastropoda, which qualitatively constituted a representative part of the community (14 taxa as a whole), attained comparatively high percentages only at station $\mathrm{D}(1.1 \%$ and $3.7 \%$ respectively). The species of gastropods previously identified by Nocentini (1973) were found again, except all Planorbidae (Hippeutis

Table 2.- Total number of specimens collected at each station and at the three depth intervals (expressed as annual averages) and respective percentage values of the zoological groups at the sampling stations. As the samples are semi-quantitative. the numbers of specimens have reported as comparative data.

Número total de individuos recolectados en cada estación y en los tres niveles de profundidad (expresado en medias anuales) y porcentajes respectivos de los grupos zoológicos cn los puntos de muestreo. Puesto que las muestras son semicuantitativas, el numero de individuos tienen valor comparativo.

\begin{tabular}{|c|c|c|c|c|c|c|c|c|}
\hline \multirow[b]{2}{*}{ Stations } & \multicolumn{4}{|c|}{ Number of specimens } & \multicolumn{4}{|c|}{$\%$} \\
\hline & $A$ & $B$ & $C^{\prime}$ & $D$ & $A$ & $\boldsymbol{B}$ & $\mathrm{C}$ & $D$ \\
\hline Hydroida & 143 & 24 & 682 & 979 & 0.2 & 0.05 & 1.3 & 3.2 \\
\hline Turbellaria & 168 & 157 & 230 & 289 & 0.3 & 0.3 & 0.5 & 0.9 \\
\hline Ncmatoda & 1196 & 443 & 1798 & 3810 & 1.8 & 0.9 & 3.5 & 12.5 \\
\hline Oligochaeta & 6076 & 119 & 274 & 153 & 9.4 & 0.2 & 0.5 & 0.5 \\
\hline Cladocera & 16215 & 14946 & 12117 & 9040 & 25.0 & 29.0 & 23.3 & 29.6 \\
\hline Copepoda & 39178 & 33574 & 34879 & 13969 & 60.4 & 65.2 & 67.1 & 45.7 \\
\hline Ostracoda & 26 & - & 16 & 3 & 0.04 & - & 0.03 & 0.009 \\
\hline Isopoda & 1 & 1 & 10 & 1 & 0.0008 & 0.001 & 0.02 & 0.004 \\
\hline Amphipoda & - & 2 & - & - & - & 0.003 & - & - \\
\hline Decapoda & 85 & 118 & 74 & 34 & 0.1 & 0.2 & 0.1 & 0.1 \\
\hline Ephemeroptera & 143 & 78 & 297 & 307 & 0.2 & 0.2 & 0.6 & 1.0 \\
\hline Odonata & 2 & 7 & 5 & 3 & 0.002 & 0.01 & 0.009 & 0.01 \\
\hline Heteroptera & - & 1 & 8 & - & - & 0.001 & 0.01 & - \\
\hline D. Chironomidac & 1213 & 1517 & 1256 & 522 & 1.9 & 2.9 & 2.4 & 1.7 \\
\hline D. Ceratopogonidae & - & 3 & 1 & 2 & - & 0.006 & 0.0005 & 0.004 \\
\hline Diptera alia & 1 & 5 & 5 & - & 0.0008 & 0.009 & 0.009 & - \\
\hline Trichoptera & 18 & 13 & 73 & 6 & 0.02 & 0.02 & 0.1 & 0.02 \\
\hline Lcpidoptera & 6 & 3 & 1 & 1 & 0.009 & 0.005 & 0.002 & 0.002 \\
\hline Hydracarina & 201 & 175 & 128 & 328 & 0.3 & 0.3 & 0.2 & 1.1 \\
\hline Gastropoda & 237 & 298 & 140 & 1125 & 0.4 & 0.6 & 0.3 & 3.7 \\
\hline Total & 64908 & 51484 & 51994 & 30567 & & & & \\
\hline
\end{tabular}


Table 3.- List of the identified taxa and percentage values (calculated on the annual mean abundances) at the sampling stations. * Genera and species not identified due to an accidental loss of material; $1 \mathrm{~mm}$. Tub. = immature Tubificids).

Lista de los taxones identificados y porcentajes (calculados sobre las abundancias medias anuales) en los puntos de muestreo.

* Géneros y especies no identificados por perdida accidental de material; imm. Tub. = l'ubificidos immaduros.

\begin{tabular}{|c|c|c|c|c|}
\hline Stations & $A$ & $B$ & $C$ & $D$ \\
\hline \multicolumn{5}{|l|}{ Hydroida } \\
\hline Hydra sp & 0.2 & 0.05 & 1.3 & 3.2 \\
\hline \multicolumn{5}{|l|}{ Turbellaria } \\
\hline Turbellaria undet. & 0.07 & 0.002 & - & 0.03 \\
\hline Dugesia tigrina (Girard) & 0.2 & 0.3 & 0.4 & 0.9 \\
\hline Polycelis sp. & - & 0.001 & - & - \\
\hline \multicolumn{5}{|l|}{ Ncmatoda } \\
\hline Plectus parvus Bastian & - & 0.02 & - & 0.005 \\
\hline Ethmolaimus pratensis De Man & 1.7 & 0.7 & 2.9 & 7.4 \\
\hline Tobrilus helveticus (Hofmanner) & 0.007 & 0.07 & 0.2 & 0.04 \\
\hline Tobrilus gracilis (Bastian) & - & & 0.006 & - \\
\hline Dorylaimus asymphydorus Andrassy & - & - & - & 5.0 \\
\hline Mesodorylaimus sp. & 0.006 & 0.07 & 0.4 & 0.004 \\
\hline Laymidorus sp. & 0.01 & - & & \\
\hline Paractinolaimus macrolaimus (De Man) & 0.2 & & & \\
\hline \multicolumn{5}{|l|}{ Oligochaeta } \\
\hline Chaetogaster diaphanus (Gruithuiscn) & - & 0.006 & - & - \\
\hline Chaetogaster diastrophus (Gruithuisen) & 0.02 & 0.03 & 0.009 & 0.008 \\
\hline Chaetogaster limnaei Von Bacr & - & 0.005 & - & \\
\hline Amphichaeta leydigii Tabucr & 0.0006 & 0.002 & - & \\
\hline Nais communis Piguet & 1.2 & 0.007 & 0.01 & 0.008 \\
\hline Nais christinae Kasparzak & - & 0.01 & - & 0.1 \\
\hline Nais variabilis Piguet & 4.6 & 0.04 & 0.1 & 0.03 \\
\hline Nais simplex Piguet & 3.4 & 0.008 & 0.1 & 0.04 \\
\hline Nais barbata (Müller) & 0.006 & - & - & 0.007 \\
\hline Pristina aequiseta Bourne & 0.02 & 0.02 & 0.03 & 0.004 \\
\hline Pristinu longiseta Ehrenberg & & 0.002 & - & 0.008 \\
\hline Pristina foreli Piguet & & 0.01 & & \\
\hline Pristina sp. & - & 0.005 & & - \\
\hline Stylaria lacustris (L.) & 0.008 & - & 0.04 & 0.2 \\
\hline Dero digitata (Miiller) & 0.02 & 0.04 & 0.1 & 0.008 \\
\hline Aulodrilus pluriseta (Piguet) & 0.003 & & & - \\
\hline Psammoryctes albicola (Michaelsen) & 0.0002 & - & - & 0.008 \\
\hline Imm. Tub. with hair chaetac & 0.03 & 0.01 & 0.08 & 0.02 \\
\hline Imm. Tub. without hair chaetae & 0.01 & 0.02 & - & \\
\hline \multicolumn{5}{|l|}{ Cladocera } \\
\hline Daphnia sp. & 0.3 & 0.5 & 0.09 & 0.3 \\
\hline Ceriodaphnia pulchella Sars & 1.7 & 1.8 & - & 0.5 \\
\hline Simocephalus vetulus (O.F. Miiller) & 0.9 & 4.7 & 4.9 & 6.6 \\
\hline Simocephalus serrulatus (Koch) & 0.2 & 1.1 & - & - \\
\hline Bosmina longirostris (O.F. Müller) & - & 0.001 & - & 0.2 \\
\hline llyocryptus sordidus Lièvin & 0.002 & 0.008 & - & - \\
\hline Eurycercus lamellatus (O.F. Müller) & 1.8 & 1.8 & 4.9 & 6.8 \\
\hline Acroperus harpae (Baird) & 5.6 & 4.3 & 2.2 & 10.3 \\
\hline Alona affinis (Leydig) & 9.9 & 0.8 & 5.5 & 0.6 \\
\hline Leydigia acanthocercoides (Fischcr) & - & 0.003 & - & \\
\hline Chydorus sphaericus (O.F. Müller) & 4.6 & 13.6 & 5.5 & 2.8 \\
\hline Alonella exigua (Lilljeborg) & - & 0.4 & 0.2 & 1.5 \\
\hline
\end{tabular}


Table 3.- Continuation

\begin{tabular}{|c|c|c|c|c|}
\hline Stations & $A$ & $B$ & $C$ & $D$ \\
\hline \multicolumn{5}{|l|}{ Copepoda } \\
\hline Macrocyclops albidus (Jurine) & 19.3 & 29.0 & 16.8 & 19.7 \\
\hline Eucyclops serrulatus (Fischer) & 11.0 & 15.1 & 14.3 & 10.5 \\
\hline Eucyclops macruroides (Lilljeborg) & 5.8 & 16.3 & 10.2 & 5.9 \\
\hline Paracyclops affinis (G.O. Sars) & 0.2 & 0.3 & - & - \\
\hline Cyclops abyssorum Sars & 23.0 & 3.7 & 24.1 & 0.8 \\
\hline Mesocyclops leuckarti (Claus) & 0.6 & 0.8 & 1.1 & 8.8 \\
\hline Harpacticoida & 0.6 & 0.02 & 0.6 & 0.04 \\
\hline \multicolumn{5}{|l|}{ Ostracoda } \\
\hline Ostracoda undet. & 0.04 & & & - \\
\hline Cypridopsis vidua (O.F. Müller) & - & - & - & 0.008 \\
\hline Limnocythere inopinata (Baird) & - & - & 0.004 & - \\
\hline Candona $\mathbf{s p .}$ & - & - & 0.03 & - \\
\hline \multicolumn{5}{|l|}{ Isopoda } \\
\hline Proasellus coxalis (Dollfus) & 0.0008 & 0.001 & 0.02 & 0.003 \\
\hline \multicolumn{5}{|l|}{ Amphipoda } \\
\hline Echinogammarus sp. & & 0.003 & - & \\
\hline \multicolumn{5}{|l|}{ Decapoda } \\
\hline Palaemonetes antennarius (Milne Edwards) & 0.1 & 0.2 & 0.1 & 0.1 \\
\hline \multicolumn{5}{|l|}{ Ephemeroptera } \\
\hline Ephemeroptera undet. & 0.07 & 0.05 & 0.06 & 0.2 \\
\hline Baetidae & 0.03 & 0.02 & 0,03 & 0.09 \\
\hline Centroptilum & 0.05 & 0.04 & 0.1 & 0.3 \\
\hline Cloeon & 0.02 & 0.04 & 0.05 & 0.08 \\
\hline Procloeon & 0.01 & 0.004 & 0.04 & 0.04 \\
\hline Caenis & 0.02 & 0.0008 & 0.3 & 0.2 \\
\hline \multicolumn{5}{|l|}{ Odonata } \\
\hline Pyrrhosoma nimphula (Sulzer) & 0.002 & 0.01 & 0.009 & 0.01 \\
\hline \multicolumn{5}{|l|}{ Heteroptera } \\
\hline Corixinae & - & 0.001 & 0.01 & - \\
\hline Micronecta & - & - & 0.002 & - \\
\hline \multicolumn{5}{|l|}{ Diptera Chironomidae } \\
\hline Psectrocladius psilopterus $\mathbf{g r}$ & 0.9 & 0.3 & 0.8 & 0.4 \\
\hline Cricotopus & 0.01 & 0.1 & 0.05 & \\
\hline Hydrobaenus & 0.001 & 0.003 & 0.009 & - \\
\hline Tanytarsus & 0.3 & 1.2 & 0.6 & 0.2 \\
\hline Paratanytarsus & - & - & 0.001 & - \\
\hline Procladius & 0.03 & 0.07 & 0.1 & 0.05 \\
\hline Larsia & 0.2 & 0.06 & 0.2 & 0.2 \\
\hline Labrutrdinia & 0.2 & 0.2 & 0.08 & 0.7 \\
\hline Nilothauma & & 0.003 & - & \\
\hline Cryptochironomus & - & 0.006 & - & - \\
\hline Pseudochironomus & 0.003 & 0.02 & 0.03 & 0.06 \\
\hline Parachironoтиs & 0.001 & 0.001 & 0.03 & 0.1 \\
\hline Chironomus & 0.03 & 0.07 & - & 0.01 \\
\hline Polypedilum laetum gr. & 0.008 & 0.006 & - & \\
\hline Polypedilum nubeculosum gr. & 0.0004 & - & - & \\
\hline Polypedilum bicrenatum gr. & 0.0004 & 0.006 & 0.03 & - \\
\hline Cryptocladopelma laccophila gr. & 0.004 & 0.3 & 0.03 & 0.007 \\
\hline Cryptocladopelma lateralis gr. & 0.001 & - & 0.003 & - \\
\hline Glyptotendipes & 0.03 & 0.02 & - & \\
\hline Microtendipes & 0.01 & 0.1 & 0.04 & - \\
\hline Paratendipes & 0.03 & 0.1 & 0.07 & 0.03 \\
\hline Dicrotendipes & 0.06 & 0.1 & 0.3 & 0.008 \\
\hline Phaenopsectra & 0.006 & 0.03 & - & - \\
\hline
\end{tabular}


Table 3.- Continuation

\begin{tabular}{|c|c|c|c|c|}
\hline Stations & $A$ & $\boldsymbol{B}$ & $C$ & $D$ \\
\hline Diptera Ceratopogonidae & - & 0.006 & 0.0005 & 0.004 \\
\hline Diptera alia & 0.0008 & 0.009 & 0.009 & - \\
\hline \multicolumn{5}{|l|}{ Trichoptera } \\
\hline Ecnomus tenellus (Rambur) & 0.03 & 0.02 & 0.1 & 0.01 \\
\hline Agrypnra varia (Fabricius) & 0.0002 & - & - & - \\
\hline Tinodes sp. & - & - & 0.006 & 0.0005 \\
\hline \multicolumn{5}{|l|}{ Lepidoptera } \\
\hline Petrophila sp. & 0.009 & 0.005 & 0.001 & 0.002 \\
\hline \multicolumn{5}{|l|}{ Hydracarina } \\
\hline Limnesia maculata (O.F. Müller) & 0.1 & 0.2 & 0.2 & 0.5 \\
\hline Neumania spinipes (O.F. Müller) & 0.1 & 0.07 & * & 0.5 \\
\hline Mideopsis orbicularis (O.F. Müller) & 0.0008 & 0.0004 & * & 0.02 \\
\hline Forelia sp. & - & 0.03 & * & 0.009 \\
\hline Lebertia sp. & 0.02 & - & * & - \\
\hline Arrhenurus sp. & 0.006 & 0.03 & * & 0.05 \\
\hline Hydrochoreutes sp. & 0.05 & 0.02 & $*$ & 0.04 \\
\hline Unionicola sp. & - & 0.007 & * & - \\
\hline \multicolumn{5}{|l|}{ Gastropoda } \\
\hline Physa acuta (Draparnaud) & 0.04 & 0.05 & 0.01 & 0.003 \\
\hline Lymnaea auricularia (L.) & 0.0004 & & 0.01 & - \\
\hline Acroloxus lacustris (L.) & 0.0002 & & 0.002 & 0.001 \\
\hline Theodoxus fluviatilis (L.) & 0.0004 & 0.003 & 0.002 & 0.001 \\
\hline Valvata piscinalis (Müller) & 0.02 & 0.1 & 0.02 & 0.9 \\
\hline Bithynia tentaculata (L.) & 0.2 & 0.4 & 0.2 & 2.3 \\
\hline Hydrobioidea & 0.03 & 0.04 & 0.04 & 0.5 \\
\hline
\end{tabular}

complanatus, Armiger crista and Anisus spirorbis), whilst Hydrobioidea represent a new record in the lake.

\section{Distribution}

A high number of taxa were observed mainly at stations A and B (841110 and 891110 respectively). Relatively high values of qualitative similarity (QS, table 4) revealed a fairly homogeneous distribution of taxa at the stations. A clear reduction of taxa belonging to insects was observed only at station D (table 5). The values of quantitative similarity (PSc, table 4) were rather high between stations A-C (77.6) and secondly B-C (64.9), whilst station D showed lower values of PSc. This situation reflects some qualitative and quantitative differences observed in the fauna composition at station D. As mentioned above, this zone of the lake was characterized qualitatively by a lower number of insect taxa, and quantitatively by considerable presence of Nematoda, lower predomi-
Table 4.- Matrices of qualitative (QS) and quantitative (PSc) similarity between stations. PSc have been calculated on the annual mean abundances.

Matriz de similaridad cualitativa (QS) y cuantitativa (PSc) entre las estaciones de muestreo. El PSc se ha calculado a partir de las abundancias medias anuales.

\begin{tabular}{lccccccccc}
\hline$(Q S)$ & $\boldsymbol{A}$ & $\boldsymbol{B}$ & $\boldsymbol{C}$ & $\boldsymbol{D}$ & $(P S C)$ & $\boldsymbol{A}$ & $B$ & $\mathrm{C}$ & $\boldsymbol{D}$ \\
$\mathrm{A}$ & - & 75.1 & 77.1 & 74.3 & & - & 58.4 & 77.6 & 53.7 \\
$\mathrm{~B}$ & & - & 74.6 & 70.8 & & & - & 64.9 & 56.3 \\
$\mathrm{C}$ & & & - & 79.4 & & & & - & 57.8 \\
$\mathrm{D}$ & & & & - & & & & & - \\
\hline
\end{tabular}

nance of Crustacea (principally Copepoda and Cladocera) and higher abundances of Hydroida, Hydracarina and Gastropoda.

The bathymetric distribution of taxa (table 5) showed a higher species richness in the marginal area $(0-3 \mathrm{~m})$ due, for the most part, to the number of insect taxa, which decreased from 34 to 23 taxa at greater depths. Diversity and evenness va- 
Table 5.- Distribution of the number of taxa at the depth intervals and at the four stations. "Hydracarina not identified. Distribución del número de taxones en los distintos intervalos de profundidad cn cuatro estaciones de muestreo. * Hidrácaros no identificados.

\begin{tabular}{|c|c|c|c|c|c|c|c|c|}
\hline & \multicolumn{4}{|c|}{ Number of taxa } & \multicolumn{4}{|c|}{ Number of taxa } \\
\hline & Total & $0-3 \mathrm{~m}$ & $3-6 m$ & $6.10 \mathrm{~m}$ & $A$ & $B$ & $\mathrm{C}$ & $D$ \\
\hline Hydroida & 1 & 1 & 1 & I & 1 & 1 & 1 & 1 \\
\hline Turbellaria & 3 & 2 & 2 & 3 & 2 & 3 & 1 & 2 \\
\hline Nematoda & 8 & 5 & 7 & 4 & 5 & 4 & 4 & 5 \\
\hline Oligochaeta & 19 & 15 & 11 & 11 & 13 & 15 & 8 & 12 \\
\hline Cladocera & 12 & 9 & 8 & 11 & 9 & 12 & 7 & 9 \\
\hline Copepoda & 7 & 7 & 7 & 6 & 7 & 7 & 6 & 6 \\
\hline Ostracoda & 4 & 2 & 3 & 2 & 1 & - & 2 & 1 \\
\hline Isopoda & 1 & 1 & 1 & - & 1 & 1 & 1 & 1 \\
\hline Amphipoda & 1 & - & 1 & - & - & 1 & - & - \\
\hline Decapoda & 1 & 1 & 1 & 1 & 1 & 1 & 1 & 1 \\
\hline Ephemeroptera & 6 & 6 & 6 & 5 & 6 & 6 & 6 & 6 \\
\hline Odonata & 1 & 1 & 1 & 1 & 1 & 1 & 1 & 1 \\
\hline Heteroptera & 2 & 2 & - & - & - & 1 & 2 & - \\
\hline D. Chironomidae & 23 & 19 & 18 & 16 & 20 & 20 & 16 & 11 \\
\hline D. Ceratopogonidae & 1 & 1 & 1 & - & - & 1 & 1 & 1 \\
\hline Diptera alia & 1 & 1 & 1 & - & 1 & 1 & 1 & - \\
\hline Trichoptera & 3 & 3 & 2 & 1 & 2 & 1 & 2 & 2 \\
\hline Lepidoptera & 1 & 1 & 1 & - & 1 & 1 & 1 & 1 \\
\hline Hydracarina & 8 & 6 & 8 & 7 & 6 & 7 & $1^{*}$ & 6 \\
\hline \multirow[t]{2}{*}{ Gastropoda } & 7 & 7 & 7 & 5 & 7 & 5 & 7 & 6 \\
\hline & 110 & 90 & 87 & 74 & 84 & 89 & 69 & 72 \\
\hline
\end{tabular}

lues also showed a clear tendency to a progressive decrease with depth, particularly between the depth intervals $0-3$ and 3-6 $\mathrm{m}$ (table 6).

Therefore, these differences showed a more complex and rich community in marginal areas. This phenomenon can be considered typical of wide littoral areas, weak bottom slope and stable water level. In fact, a narrow, steep littoral belt seems to cause higher similarity (in species richness and diversity) between different depth intervals (Mastrantuono, in prep.), whilst water level fluctuations induce the opposite trend, characterized by an increase in species richness, diversity and abundances at greater depths (GrIMÅs, 1965; Mastrantuono, 1987).

\section{DISCUSSION}

The analysis of the biocoenosis associated with submerged macrophytes in the examined zones of

Table 6.- Number of taxa, Shannon index (H) and evenness index (e) (calculated on the annual mean abundances) at the depth intervals of the sampling stations.

Numero de taxones, índice de Shannon (H) e índice de uniformidad (calculado a partir de las abundancias medias anuales) en los distintos intervalos de profundidad de las estaciones de muestreo.

\begin{tabular}{|c|c|c|c|c|c|c|c|c|c|c|c|c|}
\hline & \multicolumn{3}{|c|}{$A$} & \multicolumn{3}{|c|}{$B$} & \multicolumn{3}{|c|}{ C } & \multicolumn{3}{|c|}{$D$} \\
\hline & taxa & $H$ & $e$ & taxa & $H$ & $e$ & taxa & $H$ & $e$ & $\operatorname{tax} a$ & $H$ & $e$ \\
\hline $0-3 \mathrm{~m}$ & 67 & 3.6 & 0.59 & 58 & 3.6 & 0.61 & 56 & 3.3 & 0.57 & 48 & 3.9 & 0.69 \\
\hline $3.6 \mathrm{~m}$ & 56 & 3.2 & 0.55 & 51 & 3.0 & 0.52 & 53 & 2.9 & 0.50 & 50 & 3.6 & 0.62 \\
\hline $6-10 \mathrm{~m}$ & 55 & 3.2 & 0.55 & 56 & 2.9 & 0.50 & 41 & 2.6 & 0.49 & 50 & 3.5 & 0.62 \\
\hline
\end{tabular}


the lake revealed a community which was qualitatively rich and quantitatively rather abundant. A total of 110 taxa were identified, well distributed within numerous zoological groups and largely represented in all stations.

The fauna was mainly composed of widely diffused and euryoecious taxa. This aspect, which generally characterizes the community associated with submerged macrophytes in lakes, has always raised difficulties in the identification of bioindicator species and of structural parameters of the community which would be useful to water quality evaluation. Nevertheless, in this regard particular attention has been addressed principally to Cladocera and Gastropoda, so far representing the main organisms to which a certain role can be assigned as bioindicators in littoral lacustrine zones (Gliwicz, 1969; Clarke, 1979; Mouthon, $\left.1981 ; \emptyset_{\text {KLAND, }} 1983\right)$

The importance of microfilterer cladocerans in a trophic evaluation may be related to their ability to use minute particles (tripton and seston) as food source. An increase in organic matter in the water generally induces both a diminution of cladoceran species (due to the disappearance of macrofilterers) and an increase in density of microfilterers (GLIwICZ, 1969). This phenomenon clearly emerged in the polluted Lake Nemi (MASTRANTUONO, 1986), where only three littoral cladocerans were found and Chydorus sphaericus alone accounted for most of the total fauna. On the contrary, Lake Vico was characterized, like the oligo-mesotrophic Lake Campotosto, by a high number of cladoceran species (12), for the most part macrofilterers (primarily S. vetulus, $S$. serrulatus, E. lamellatus, Daphnia sp. and secondarily $A$. affinis and $A$. harpae) which showed notable abundance at all stations. Moreover, relatively low percentages of the microfilterer Chydorus sphaericus (range: 2.8-13.6) were observed in the lake, particularly at station D. The relative abundance of this species seems to constitute a indicative parameter of trophic level, considering the satisfactory agreement with indications founded on the analysis of other parameters such as macrophyte composition, chemical data and diversity values (Mastrantuono, 1986, 1987).

According to a classification of sensitivity to pollution proposed by MouTHON (1981) for gastropods, all taxa found in Lake Vico, excluding
Table 7.- Values of some parameters of the community at the sampling stations. $\mathrm{H}$ and e have been calculated on cumulative data of the three depth intervals.

Valores de algunos de los parametros de la comunidad en los puntos de muestreo. H y e se han calculado en base a datos acumulativos de los tres intervalos de profundidad.

\begin{tabular}{lrrrr}
\hline & \multicolumn{4}{c}{ Stations } \\
\cline { 2 - 5 } & \multicolumn{1}{c}{$A$} & \multicolumn{1}{c}{$B$} & \multicolumn{1}{c}{$C$} & \multicolumn{1}{c}{$D$} \\
\hline Colonized belt (m) & $0-8$ & $0-8$ & $0-8$ & $0-10$ \\
Species richness & 84 & 89 & 69 & 72 \\
Chydorus sphaericus (\%) & 4.6 & 13.6 & 5.5 & 2.8 \\
Hydrobioidea (\%) & 0.03 & 0.04 & 0.04 & 0.5 \\
Total fauna & 64908 & 51484 & 51994 & 30567 \\
Diversity ([I) & 3.6 & 3.3 & 3.5 & 3.9 \\
Evenness (c) & 0.57 & 0.52 & 0.57 & 0.65 \\
\hline
\end{tabular}

Hydrobioidea, belong to pollutant-resistant levels. Hydrobioidea, considered sensitive to environmental modifications, have so far been found in Central Italy only in the largest lakes (Lake Bracciano and Bolsena; Nocentini, 1973). In Lake Vico very low percentages occurred at stations A, $\mathrm{B}$ and $\mathrm{C}$, close to the cultivated lands, and only at station $\mathrm{D}$ was a more conspicuous presence of these organisms $(0.5 \%)$ evidenced.

On the ground of the results obtained in this study the community structure in the examined areas of Lake Vico is characterized by: a) high species richness (110 taxa), b) high number of cladoceran species $(12), \mathrm{c})$ relatively low percentages of the microfilterer Chydorus sphaericus, d) medium-high values of relative abundance of total fauna, e) coexistence of tolerant molluscan species with others sensitive to environmental modifications, f) relatively high values of diversity (range: 2.6-3.9) and evenness (range: 0.49-0.69). These parameters taken together can be considered indicative of a satisfactory environmental quality in the littoral zones examined. Such an evaluation is in good accordance with the indications of oligo-mesotrophy founded on macrophyte composition and chemical data.

So, the influence of the cultivations still appears to be reduced in littoral waters, also at the stations located close the cultivated lands $(\mathrm{A}, \mathrm{B}, C)$, where the sandy sediments appeared clearly affected by an organic enrichment (MastrantuONo \& LA RoccA, 1988). This different result can be easily explained considering that the zoobenthos associated with submerged macrophytes generally 
shows a much higher degree of resistance (homeostasis) to environmental modifications than communities associated to littoral sediments, following the high dispersion of physico-chemical factors in the waters.

Nevertheless, the comparison of the values of the more significant parameters at the stations (table 7) showed the different situation of station $\mathrm{D}$, characterized by lower percentages of Chydorus sphaericus, low values of total fauna, high values of diversity and evenness and higher abundances of Hydrobioidea. All these characteristics are indicative of a low trophic level in this zone of the lake, according to the results obtained in the study of the zoobenthos living in sandy shores.

The differences observed in the lake between the station unaffected by the surrounding cultivations and the remaining ones confirm the importance of the above mentioned parameters of the community in water quality evaluation, in agreement with analogous results obtained in previous studies. These elements encourage further analyses specifically addressed to those particular aspects of this biocoenosis which can be used for a more accurate evaluation of the environmental quality in littoral lacustrine zones.

\section{ACKNOWLEDGEMENTS}

This study was partially supported by a grant of «Stabilimento Ittiogenico, Regione Lazio», Italy. I am particularly indebted to Dr. E. Gelosi (director of the «Stabilimento Ittiogenico») for his assistance in the organization of the field-work, to Dr. G. Nicotra, who kindly supplied physicochemical data and to Dr. C. La Rocca for her technical help.

\section{REFERENCES}

Barbanti, L., 1969. Lago di Vico: rilevamento batimetrico e note geomorfologiche. Mem. Ist. Ital. Idrobiol., 25: 117-139.

Barbanti, L., G. Bonomi, A. Carollo, G. Chiaudani, I. Ferrari, M. Gerletti, A.M. Nocentini, D. Ruggiu \& L. TONOLLI, 1971. Limnologia ed ecologia dei laghi di Bolsena, Bracciano, Trasimeno e Vico: situazione attuale e prevedibili conseguenze derivanti da una loro utilizzazione multipla. Ist. Ital. Idrobiol., Verbania Pallanza, 263 pp.

BigGS, B.J.F. \& T.J. Malthus, 1982. Macroinvertebrates associated with various aquatic macrophytes in the backwaters and lakes of the upper Clutha Valley, New Zealand. New Zeal. J. Mar. Freshwat. Res., 16: 81-X8
Brown, C.L.. T.P. Poe, J.R.P. French III \& D.W. SchloesSER, 1988. Relationships of phytomacrofauna to surface area in naturally occurring macrophyte stands. J.N. Am. Benthol. Soc., 7: 129-139.

ClARKL, A.H., 1979. Gastropods as indicators of trophic lake stages. The Nautilus, 94: 138-142.

GLIwICZ, Z.M., 1969. Studies on the feeding of pelagic zooplankton in lakes with varying trophy. Ekol. Pol., 17: 1-45.

GRIMÅs. U., 1965. The short-term effect of artificial water-level fluctuations upon the littoral fauna of Lake Kultsjon, northern Sweden. Inst. Freshwai. Res. Drotiningholm, report nr. 46, 5-21.

LACHAVANNE, J.B., 1985. The influence of accelerated eutrophication on the macrophytes of Swiss lakes: Abundance and distribution. Verh. Internat. Verein. Limnol., 22: 2950-2955.

MARGAlef, R., 1957. La teoria de la information en ecologia. Mem. Real Acad. Ciencias y Artes de Barcelona, 32: 373-449.

Mastrantuono, L, 1986. Community structure of the zoobenthos associated with submerged macrophytes in the eutrophic Lake Nemi. Boll. Zool., 53: 41-47.

MASTRANTUONO, L., 1987. Invertebrate community in the littoral-regulated area of a hydroelectric lake-reservoir (Lake Campotosto, Central Italy). Riv. Idrobiol., 26: 17-32.

Mastrantuono, L. \& C. La Rocca, 1988. The invertebrate fauna in sandy shores of Lake Vico (Italy): its use in a trophic evaluation of littoral sediments. Ecol. Mediter., 14: 121-129.

Mouthon, J., 1981. Les mollusques et la pollution des eaux douces: ébauche d'une gamme de polluosensibilité des espéces. Bij. Dierkol., 51: 250-258.

NocentinI, A.M., 1973. La fauna macrobentonica litorale e sublitorale dei laghi di Bolsena, Bracciano e Vico (Italia Centralc, Lazio). Mem. Ist. Ital. Idrobiol., 30: 97-148.

O.E.C.D. (Organization for Economic Cooperation and Development), 1982. Eutrophication of waters. Monitoring, assessment and control. O.E.C.D., Paris.

$\emptyset_{K L A N D}$, J., 1983. Factors regulating the distribution of freshwater snails (Gastropoda) in Norway. Malacologia, 24: 277-288.

OZIMEK, T. \& U. SIKORSKA, 1976. Influence of municipal sewage on the littoral biocoenosis. In: Pieczynska E. (ed.) Selected problems of lake littoral ecology. Univ. of Warsaw., 238 pp.

PIECZYŃSKI, E., 1973. Experimentally increased fish stock in the pond type Lake Warniak. XII. Numbers and biomass of the fauna associated with macrophytes. Ekol. Pol., 21: 596-609.

Pielov, E.C., 1966. Species-diversity and pattern-diversity in the study of ecological succession. J. theoret. biol., 10 : 370-383.

RENKonen, O., 1938. Statistish-okologische Untersuchungen über die terrestrische Kafferwelt der finnischen Bruchmoore. Ann. Zool. Soc. Zool. Bot. Fenn. Vanamo, 6: 1-231.

SøRENSEN, T., 1948. A method of stabilizing groups of equivalent amplitude in plant sociology based on the similarity of species content and its application to analysis of the vegetation on Danish commons. Biol. Skr., 5: 1-34.

SosZKA, G.J., 1975. Ecological relations between invertebrates and submerged macrophytes in the lake littoral. Ekol. Pol., 23: 393-415. 